\title{
Spitzer Space Telescope Observations of Low Mass X-ray Binaries
}

\author{
Stefanie Wachter \\ Spitzer Science Center, California Institute of Technology, 1200 E. California Blvd. \\ Pasadena, CA 91125
}

\begin{abstract}
We present preliminary results from our archival Spitzer Space Telescope program aimed at characterizing the mid-IR properties of compact objects, both isolated and in binary systems, i.e. white dwarfs, X-ray binaries, cataclysmic variables, and magnetars. Most of these sources are too faint at mid-IR wavelengths to be observable from the ground, so this study provides the very first comprehensive look at the mid-IR emission of these objects. Here we present our results for the low mass X-ray binaries. We considered all of the systems listed in the most recent catalog of Liu et al. (2007) that have known optical counterparts. The particular goals of our projects encompass: to establish the mid-IR spectral energy distribution, to search for the signatures of jets, circumbinary disks, low mass or planetary companions and debris disks, and to study the local environment of these sources.
\end{abstract}

\section{Introduction}

We have searched the Spitzer Space Telescope archive (with Leopard) for observations covering the positions of all low mass X-ray binaries with known optical counterparts listed in Liu et al. (2007). Table 1 details the data available for each source as of Oct 1, 2007. Note that not all of the data listed in the table are public at the time of this writing. In Table 2 we provide some preliminary results as to whether a particular source is detected or not, together with published Spitzer measurements of LMXBs. In this context, (f) denotes faint, (ff) very faint, in a most subjective fashion. (s) indicates that the source is saturated. The Table is incomplete since we have not yet investigated all the targets that were covered with Spitzer (according to Table 1). So far, we have found 14 sources in addition to the eight published Spitzer detections of LMXBs.

Acknowledgments. The research described in this poster was carried out, in part, at the Jet Propulsion Laboratory, California Institute of Technology, and was sponsored by the National Aeronautics and Space Administration through a grant to the PI. We made use of data products from the 2 Micron All Sky Survey, which is a joint project of the University of Massachusetts and the Infrared Processing and Analysis Center/California Institute of Technology, funded by the National Aeronautics and Space Administration and the National Science Foundation. It also utilized NASA's Astrophysics Data System Abstract Service and the SIMBAD database operated by CDS, Strasbourg, France. 
Table 1. LMXBs observed by the Spitzer Space Telescope

\begin{tabular}{|c|c|c|c|c|}
\hline Name $^{a}$ & Altern. Name & RA (2000) & DEC (2000) & PID/AORkey, Instr. Mode, Notes \\
\hline IGR J00291+5934 & & 002903.06 & 593419.00 & No programs found \\
\hline $4 \mathrm{U} 0042+32$ & & 004450.40 & $3301 \quad 17.00$ & No programs found \\
\hline GRO J0422+32 & & 042142.79 & 325427.10 & 40948/23365888 IRAC Mapping \\
\hline \multirow[t]{8}{*}{$2 \mathrm{~A} 0521-720$} & LMC X-2 & 052028.13 & -715735.34 & 20203/14358272 IRAC Mapping \\
\hline & & & & 20203/14370816 IRAC Mapping \\
\hline & & & & 20203/14356480 IRAC Mapping \\
\hline & & & & 20203/14371072 IRAC Mapping \\
\hline & & & & 20203/14390016 MIPS Scan Map \\
\hline & & & & 20203/14383104 MIPS Scan Map \\
\hline & & & & 20203/14383360 MIPS Scan Map \\
\hline & & & & 20203/14389760 MIPS Scan Map \\
\hline \multirow{3}{*}{$4 \mathrm{U} 0614+091$} & & 061707.40 & 090813.60 & 20224/14415872 IRAC Mapping \\
\hline & & & & 30803/19018240 IRAC Mapping \\
\hline & & & & 30803/19018496 MIPS Photometry \\
\hline \multirow[t]{4}{*}{$1 \mathrm{~A} 0620-00$} & & 062244.50 & -002044.72 & 3289/10702592 MIPS Photometry \\
\hline & & & & $3289 / 10703616$ IRAC Mapping \\
\hline & & & & 30616/18618112 IRAC Mapping \\
\hline & & & & 30616/18618624 IRAC Mapping \\
\hline EXO $0748-676$ & & 074833.80 & -674508.93 & No programs found \\
\hline $4 \mathrm{U} 0919-54$ & & 092026.95 & $\begin{array}{llll}-55 & 12 & 24.70\end{array}$ & 20224/14416128 IRAC Mapping \\
\hline 2S $0921-630$ & & 092234.73 & -631741.37 & No programs found \\
\hline XTE J0929-314 & & 092920.19 & -312303.20 & No programs found \\
\hline GRS $1009-45$ & & 101336.30 & -450432.00 & 40948/23364864 IRAC Mapping \\
\hline GS $1124-684$ & & 112626.70 & -684032.60 & 40948/23365376 IRAC Mapping \\
\hline 1A $1246-588$ & & 124939.36 & $\begin{array}{lll}-59 & 05 & 14.68\end{array}$ & No programs found \\
\hline $4 \mathrm{U} 1254-69$ & & 125737.20 & -691720.80 & No programs found \\
\hline \multirow[t]{4}{*}{$4 \mathrm{U} 1323-62$} & & 132636.31 & -620809.90 & 190/9231616 IRAC Mapping \\
\hline & & & & 190/9234176 IRAC Mapping \\
\hline & & & & 20597/15646208 MIPS Scan Map \\
\hline & & & & 20597/15657216 MIPS Scan Map \\
\hline GS $1354-64$ & & 135809.92 & -644404.90 & No programs found \\
\hline \multirow[t]{2}{*}{$4 \mathrm{U} 1456-32$} & Cen $\mathrm{X}-4$ & 145822.00 & -314008.00 & 3289/10703360 MIPS Photometry \\
\hline & & & & 3289/10704384 IRAC Mapping \\
\hline \multirow[t]{5}{*}{ 3A $1516-569$} & Cir $\mathrm{X}-1$ & 152040.90 & -571001.00 & 190/9226752 IRAC Mapping \\
\hline & & & & 190/9228032 IRAC Mapping \\
\hline & & & & 190/9331200 IRAC Mapping \\
\hline & & & & 20597/15593984 MIPS Scan Map \\
\hline & & & & 20597/15626240 MIPS Scan Map \\
\hline 1A $1524-61$ & $\operatorname{Tr} \mathrm{A} X-1$ & 152817.20 & -615258.00 & No programs found \\
\hline $4 \mathrm{U} 1543-47$ & & 154708.60 & -474010.00 & No programs found \\
\hline $4 \mathrm{U} 1543-624$ & & 154754.29 & -623405.13 & 20224/14415616 IRAC Mapping \\
\hline XTE J1550-564 & & 155058.78 & -562835.00 & No programs found \\
\hline $4 \mathrm{U} 1556-60$ & & 160102.30 & -604418.00 & No programs found \\
\hline $1 \mathrm{E} 1603.6+2600$ & & 160545.82 & 255145.10 & No programs found \\
\hline \multirow[t]{5}{*}{$4 \mathrm{U} 1608-52$} & & 161243.00 & -522523.00 & 191/9329664 IRAC Mapping \\
\hline & & & & 191/9334784 IRAC Mapping \\
\hline & & & & 20597/15606016 MIPS Scan Map \\
\hline & & & & 20597/15653632 MIPS Scan Map \\
\hline & & & & 30570/20252928 IRAC Mapping \\
\hline \multirow[t]{3}{*}{ H $1617-155$} & Sco $\mathrm{X}-1$ & 161955.07 & -153824.80 & 20462/14727168 IRAC Mapping \\
\hline & & & & 20462/14727424 MIPS Photometry \\
\hline & & & & 20462/14727680 IRS Peakup Image \\
\hline \multirow[t]{3}{*}{$4 \mathrm{U} 1624-49$} & & 162802.83 & -491154.61 & 191/9334528 IRAC Mapping \\
\hline & & & & 20597/15610880 MIPS Scan Map \\
\hline & & & & 20597/15620608 MIPS Scan Map \\
\hline $4 \mathrm{U} 1626-67$ & & 163216.80 & -672743.00 & 20224/14415360 IRAC Mapping \\
\hline \multirow[t]{6}{*}{$4 \mathrm{U} 1630-47$} & & 163401.61 & -472334.80 & 191/9326592 IRAC Mapping \\
\hline & & & & 191/9330688 IRAC Mapping \\
\hline & & & & 20597/15645184 MIPS Scan Map \\
\hline & & & & 20597/20489984 MIPS Scan Map \\
\hline & & & & 20597/15660800 MIPS Scan Map \\
\hline & & & & 20597/20489728 MIPS Scan Map \\
\hline $4 \mathrm{U} 1636-536$ & & 164055.50 & -534505.00 & No programs found \\
\hline XTE J1650-500 & & 165000.98 & -495743.60 & No programs found \\
\hline
\end{tabular}


Table $1 . \quad$ continued

2 A $1655+353$ XTE J1701-462 MXB 1659-298 4U 1659-487

3A $1702-363$

$4 \mathrm{U} 1702-429$ $4 \mathrm{U} 1700+24$ $4 \mathrm{U} 1705-250$ XTE J1709-267 IGR J17098-3628

GRO J1719-24 XTE J1720-318 IGR J17269-4737 IGR J17285-2922

$4 \mathrm{U} 1728-16$ $4 \mathrm{U} 1728-34$

3A $1728-247$

KS $1731-260$

$4 \mathrm{U} 1735-444$ 1 H $1743-322$

IGR J17473-2721

1A $1744-361$ IGR J17497-282

EXO 1747-214

$4 \mathrm{U} 1755-33$

$4 \mathrm{U} 1758-25$

\begin{tabular}{|c|c|c|}
\hline \multirow{3}{*}{ Her $X-1$} & 165749.83 & 352032.60 \\
\hline & 170058.45 & -461108.60 \\
\hline & 170206.50 & -295644.10 \\
\hline GX339-4 & 170249.50 & -484723.00 \\
\hline
\end{tabular}

GX349+2

170544.50

$1706 \quad 15.31$ 170634.52

170814.60

170930.40

170945.93

171936.93

171958.99

172649.28

172830.00

GX $9+9$

GX $354-0$

173144.20

173157.73

GX $1+4$

173202.16

173413.47

$-260518.80$

not public not public not public not public $173858.30 \quad-442700.00$ $1746 \quad 15.57$

$\begin{array}{lll}-32 & 1401.10\end{array}$

$1748 \quad 13.15$ 174938.04

175024.52 175840.00 180108.22

$-430208.69$ 235818.60 2639 $-362758.20$

$174718.08 \quad-272038.70$

$-360757.02$

$-212519.90$ $-334827.00$
3246/10524928 MIPS Photometry 3246/10525184 MIPS Photometry 3246/10525696 MIPS Photometry 3246/10525440 MIPS Photometry 20301/14508800 IRAC Mapping 20301/14509312 MIPS Photometry 20669/14967040 IRAC Mapping 30570/20220416 IRAC Mapping 30570/21312768 IRAC Mapping 30570/21311232 IRAC Mapping

No programs found

30570/21285376 IRAC Mapping No programs found

3246/10524416 MIPS Photometry 3246/10524672 MIPS Photometry 30570/20246528 IRAC Mapping 30570/21296384 IRAC Mapping 30570/21324544 IRAC Mapping

No programs found

No programs found

No programs found

No programs found

30570/20246528 IRAC Mapping 30570/21296384 IRAC Mapping 30570/21324544 IRAC Mapping

No programs found

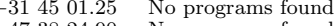

No programs found 30570/20204288 IRAC Mapping 30570/21324032 IRAC Mapping No programs found

20201/14292992 IRAC Mapping 20201/14293248 IRAC Mapping 20201/14323200 IRAC Mapping 20201/14322944 IRAC Mapping 20119/14093056 IRAC Mapping 20119/14099456 MIPS Scan Map 20119/14099712 MIPS Scan Map 20119/14090496 IRAC Mapping 20119/14099968 MIPS Scan Map 20119/14100480 MIPS Scan Map 30570/21277184 IRAC Mapping 30570/21335552 IRAC Mapping 30570/21291776 IRAC Mapping 30570/21307136 IRAC Mapping No programs found

30570/20205824 IRAC Mapping 30570/20210432 IRAC Mapping 30570/21306624 IRAC Mapping 30570/21313024 IRAC Mapping 30594/20515840 MIPS

30594/20517888 MIPS 30594/20516864 MIPS

20201/14309888 IRAC Mapping 20201/14309632 IRAC Mapping 20201/14339328 IRAC Mapping 20201/14339584 IRAC Mapping 20414/14658048 MIPS Scan Map 20414/14659072 MIPS Scan Map

No programs found

No programs found

3677/13368320 IRAC Mapping 3677/13370624 IRAC Mapping 20201/14308096 IRAC Mapping 20201/14337792 IRAC Mapping 20414/14657792 MIPS Scan Map 20414/14657792 MIPS Scan Map 20414/14658048 MIPS Scan Map
30570/20208640 IRAC Mapping No programs found 20201/14314752 IRAC Mapping 20201/14312704 IRAC Mapping 20201/14342400 IRAC Mapping 20201/14344448 IRAC Mapping 30570/21293568 IRAC Mapping 
Table $1 . \quad$ continued

GRS 1758-258

SAX J1808.4-3658 SAX J1810.8-2609 XTE J1814-338 $4 \mathrm{U} 1811-17$

$4 \mathrm{U} 1812-12$

4U 1813-14 AX J1824.5-2451 $4 \mathrm{U} 1823-00$ 2A $1822-371$
180112.40

180827.60 181044.47 $\begin{array}{llll}18 & 13 & 39.04\end{array}$ GX $13+1 \quad 18 \quad 1431.55$

181506.16

GX $17+2$ 181601.39 182430.00 182522.02 182546.80

182928.20 $4 \mathrm{U} 1905+000$ XTE J1908+094

$4 \mathrm{U} 1908+005$ GRS $1915+105$ 183957.56 185841.58 190008.65 190826.97 190853.08

Ser $\mathrm{X}-1$

$1911 \quad 16.00$

Aql X-1 1915 $\begin{array}{lll}-23 & 4749.12\end{array}$ 050209.60 223929.40 $-245513.70$ 001007.70 092304.90

003506.00 105644.76

\author{
4U 1916-05 \\ 3A $1954+319$ \\ $4 \mathrm{U} 1957+11$ \\ GS $2000+25$
}

XTE J2012+381
191847.87 195542.33

$\begin{array}{lll}19 & 5924.20\end{array}$ $\begin{array}{llll}19 & 02 & 49.58\end{array}$ $2012 \quad 37.71$ $\begin{array}{lll}-05 & 14 & 17.09\end{array}$ $\begin{array}{llll}32 & 05 & 49.10\end{array}$

$\begin{array}{llll}11 & 42 & 32.40\end{array}$

$25 \quad 14 \quad 11.30$ 381101.10
20201/14312192 IRAC Mapping 20201/14341888 IRAC Mapping

No programs found

No programs found

No programs found

146/12104192 IRAC Mapping 146/12108800 IRAC Mapping 20597/15598848 MIPS Scan Map 20597/15634944 MIPS Scan Map 20597/15632384 MIPS Scan Map 30570/21288448 IRAC Mapping $30570 / 21288448$ IRAC Mapping
$30570 / 21309440$ IRAC Mapping No programs found

40111/21745408 IRS Staring

20224/14415104 IRAC Mapping $1867 / 16040192$ MIPS Scan Map $1867 / 16040192$ MIPS Scan Map $1867 / 16040448$ MIPS Scan Map
$1867 / 16040704$ MIPS Scan Map $1867 / 16040704$ MIPS Scan Map
$1867 / 16040960$ MIPS Scan Map $1867 / 16041216$ MIPS Scan Map 1867/16041472 MIPS Scan Map $1867 / 16041728$ MIPS Scan Map 1867/16041984 MIPS Scan Map $1867 / 16042240$ MIPS Scan Map 1867/16042496 MIPS Scan Map $1867 / 16042752$ MIPS Scan Map 1867/16043008 MIPS Scan Map $1867 / 16043264$ MIPS Scan Map 1867/16043520 MIPS Scan Map 1867/16043776 MIPS Scan Map 1867/16044032 MIPS Scan Map 1867/16044288 MIPS Scan Map 1867/16044544 MIPS Scan Map 1867/16044800 MIPS Scan Map $1867 / 16045056$ MIPS Scan Map $1867 / 16045056$ MIPS Scan Map
$1867 / 16057856$ MIPS Scan Map $1867 / 16058112$ MIPS Scan Map $1867 / 16058368$ MIPS Scan Map No programs found

174/5716992 MIPS Scan Map 40948/23365120 IRAC Mapping No programs found No programs foun 187/11963904 IRAC Mapping 20597/15602688 MIPS Scan Map 20597/15614720 MIPS Scan Map

No programs found

187/11970048 IRAC Mapping 187/11973120 IRAC Mapping 3352/10831616 IRAC Mapping 3352/10832128 IRS Staring 3352/10832384 IRS Staring $3352 / 10832640$ IRS Staring $3352 / 10832640$ IRS Staring
$3352 / 10832896$ IRS Staring 3352/10831360 IRAC Mapping 3352/10831104 IRAC Mapping 3352/10831872 IRAC Mapping 20232/14420480 IRAC Mapping 20232/14420736 IRAC Mapping 20232/14420992 IRAC Mapping 20232/14420224 IRAC Mapping 20232/14421248 IRS Staring 20232/14422016 IRS Staring 20232/14421504 IRS Staring 20232/14421760 IRS Staring 20597/15599616 MIPS Scan Map 20597/15614976 MIPS Scan Map 20224/14414848 IRAC Mapping

No programs found

No programs found 40948/23365632 IRAC Mapping 20726/15055616 MIPS Photometry 20726/15055872 IRAC Mapping 
Table 1. continued

\begin{tabular}{lcrrl} 
Name $^{a}$ & Altern. Name & RA (2000) & DEC (2000) & PID/AORkey, Ins tr. Mode, Notes \\
\hline GS 2023+338 & & 202403.80 & 335203.20 & $3289 / 10703104$ MIPS Photometry \\
& & 212314.54 & -054753.20 & $\begin{array}{l}\text { No programs found } \\
\text { XTE J2123-058 }\end{array}$ \\
4U 2129+47 & & 213126.20 & 471724.00 & No programs found \\
4U 2142+38 & Cyg X-2 & 214441.20 & 381918.00 & No programs found
\end{tabular}

${ }^{a}$ nomenclature from Liu et al. (2007)

Table 2. Preliminary Results

\begin{tabular}{|c|c|c|c|c|c|c|}
\hline Name & $\begin{array}{c}\text { F3.6 } \\
(\mathrm{mJy})\end{array}$ & $\begin{array}{c}\text { F4.5 } \\
(\mathrm{mJy})\end{array}$ & $\begin{array}{c}\text { F5.8 } \\
(\mathrm{mJy})\end{array}$ & $\begin{array}{c}\text { F8.0 } \\
(\mathbf{m J y})\end{array}$ & $\begin{array}{c}\mathbf{F 2 4} \\
(\mathbf{m J y})\end{array}$ & Notes \\
\hline LMC X-2 & 0.038 & $\operatorname{yes}(f)$ & no & no & no & SAGE reliable, this work \\
\hline X0614+091 & 0.16 & 0.18 & 0.2 & 0.25 & & Migliari et al. (2006) \\
\hline \multirow[t]{2}{*}{ X0620-003 } & $\ldots$ & 0.4 & $\ldots$ & 0.29 & 0.14 & Gallo et al. (2007) \\
\hline & $\ldots$ & 0.45 & $\ldots$ & 0.25 & 0.05 & Muno et al. (2006) \\
\hline X0918-549 & no & no & no & no & no & this work \\
\hline \multirow[t]{2}{*}{$\mathrm{J} 1118+480$} & $\ldots$ & 0.069 & $\ldots$ & 0.059 & $<0.05$ & Gallo et al. (2007) \\
\hline & $\ldots$ & 0.046 & $\ldots$ & 0.045 & $<0.02$ & Muno et al. (2006) \\
\hline Cen $\mathrm{X}-4$ & . & 0.20 & $\ldots$ & 0.095 & $<0.03$ & Muno et al. (2006) \\
\hline Cir $\mathrm{X}-1$ & 19.3 & 14.9 & 12.0 & 6.6 & 6 & Glimpse reliable, MIPS24 this work \\
\hline $\mathrm{X} 1323-62$ & no & no & no & no & no & Counterpart uncertain \\
\hline $\mathrm{X} 1543-62$ & no & no & no & no & $\ldots$ & this work \\
\hline $\mathrm{X} 1608-52$ & yes(f) & yes(ff) & no & no & no & this work, very crowded \\
\hline $\mathrm{Sco} X-1$ & 10 & 7 & 4 & 1.5 & 1 & Wachter et al. (2006) \\
\hline $\mathrm{X} 1624-490$ & no & no & no & no & no & very crowded \\
\hline $\mathrm{X} 1626-67$ & yes(f) & $\operatorname{yes}(f)$ & yes(ff) & no & $\ldots$ & this work \\
\hline $\mathrm{X} 1630-47$ & no & no & no & no & no & this work, too crowded \\
\hline $1655-40$ & 5.67 & 3.90 & 3.02 & 2.40 & $<0.54-2.07$ & Migliari et al. (2007) \\
\hline GX $339-4$ & $\ldots$ & $\ldots$ & $\ldots$ & $\ldots$ & 0.135 & Tomsick et al. (2004) \\
\hline XJ1701-462 & yes & yes & yes & yes & $\ldots$ & correct ctpt? bright in all 4 channels \\
\hline GX $349+2$ & yes & yes & yes & yes (f) & $\ldots$ & this work \\
\hline IGRJ1709-362 & no & no & no & no & $\ldots$ & this work, too crowded \\
\hline GX $354-0$ & yes(f) & yes(f) & yes $(f)$ & $?$ & $\ldots$ & this work \\
\hline GX $1+4$ & yes(s) & yes & yes & yes & yes & this work, saturated ch1 \\
\hline $\mathrm{X} 1743-322$ & yes? & yes? & yes? & no & no & blend of three sources \\
\hline IGR $1747-272$ & no & no & no & no & no & too crowded \\
\hline GX 5-1 & yes & yes & no? & no & $\ldots$ & close blend of two sources \\
\hline $\mathrm{X} 1758-258$ & yes & yes & yes & no & $\ldots$ & this work \\
\hline GX $13+1$ & $(7.8)$ & 6.32 & 4.28 & 2.84 & no & GLIMPSE, no ch 1 \\
\hline $\mathrm{X} 1822-00$ & yes(f) & yes(f) & yes(ff) & no & $\ldots$ & this work \\
\hline $\mathrm{X} 1915+105$ & 4.7 & 4.9 & 4.8 & 3.1 & 28.3 & Glimpse reliable catalog, this work \\
\hline \multirow[t]{2}{*}{ V404 Cyg } & $\ldots$ & 3.3 & $\ldots$ & 1.8 & 0.4 & Gallo et al. (2007) \\
\hline & $\ldots$ & 3.0 & $\ldots$ & 1.45 & 1.53 & Muno et al. (2006) \\
\hline
\end{tabular}

\section{References}

Gallo, E. et al. 2007, ApJ, 670, 600

Liu, Q.Z., van Paradijs, J., \& van den Heuvel, E.P.J. 2007, A\&A, 469, 807

Migliari, S. et al. 2006, ApJ, 643, L41

Migliari, S. et al. 2007, ApJ, 670, 610

Muno, M., \& Mauerhan, J. 2006, ApJ, 648, L135

Tomsick, J.A. et al. 2004, BAAS, 36, 1516

Wachter, S., Bandyopadhyay, R.M., Markoff, S., \& Smale, A.P. 2006, BAAS, 38, 334 